\title{
Opposite-quadrant depth in the plane
}

\author{
Hervé Brönnimann* Jonathan Lenchner ${ }^{\dagger} \quad J^{*}$ János Pach ${ }^{\ddagger}$ \\ * Computer and Information Science, Polytechnic University, \\ Brooklyn, NY 11201, hbr@poly.edu \\ † IBM T. J. Watson Research Center, \\ Yorktown Heights, NY 10598, lenchner@us.ibm.com \\ $\ddagger$ Courant Institute, NYU, and City College, CUNY \\ 251 Mercer Street, New York, NY 10012, pach@cims.nyu.edu
}

\begin{abstract}
Given a set $S$ of $n$ points in the plane, the opposite-quadrant depth of a point $p \in S$ is defined as the largest number $k$ such that there are two opposite axis-aligned closed quadrants (NW and SE, or SW and NE) with apex $p$, each containing at least $k$ elements of $S$. We prove that $S$ has a point with opposite-quadrant depth at least $n / 8$. If the elements of $S$ are in convex position, then we can guarantee the existence of an element whose opposite-quadrant depth is at least $n / 4$. Both results are asymptotically best possible.
\end{abstract}

\section{Introduction}

Let $S$ be a set of $n$ points in the plane. Given any point $p$, the horizontal and vertical lines through $p$ define four closed quadrants $\mathrm{NW}(p), \mathrm{NE}(p), \mathrm{SE}(p)$, and $\mathrm{SW}(p)$. We define the opposite-quadrant depth $\operatorname{opp}(p, S)$ of a point $p$ with respect to $S$ as the largest number $k$ such that there are two opposite quadrants $\mathrm{NW}(p)$ and $\mathrm{SE}(p)$ or $\mathrm{NE}(p)$ and $\mathrm{SW}(p)$, each containing at least $k$ elements of $S$. In notation:

$$
\begin{aligned}
& \operatorname{opp}(p, S)= \max (\min (|\mathrm{NW}(p) \cap S|,|\mathrm{SE}(p) \cap S|), \\
&\min (|\mathrm{NE}(p) \cap S|,|\mathrm{SW}(p) \cap S|)), \\
& \operatorname{opp}(S)=\max _{p \in \mathbb{R}^{2}} \operatorname{opp}(p, S) .
\end{aligned}
$$

A point $p$ is said to be $\alpha$-deep, for some $\alpha>0$, if its opposite-quadrant depth is at least $\alpha n$. A (not necessarily unique) point of maximum opposite-quadrant depth is called a deepest point, and a point which is $\alpha$-deep is also called an $\alpha$-centerpoint. If we do not require the centerpoint to be in $S$, then there always exists a point that is $\frac{1}{4}$-deep at the intersection of the vertical and horizontal

\footnotetext{
${ }^{*}$ Research partially supported by NSF grant CCF-0133599.

${ }^{\dagger}$ Research partially supported by NSF grant CCF-0133599.

${ }^{\ddagger}$ Research partially supported by grants from NSF, NSA, OTKA, and the Israeli-US Binational Research Foundation.
} 
halving lines. In fact, $\frac{1}{4}$ is the best general bound that can be established on the maximum depth for any point in the plane, as shown by a set of $n$ points uniformly distributed in a square, a disk, or even on a circle. There are also sets with deeper points, e.g., any set of $n$ collinear points has a point of depth $\lceil n / 2\rceil$.

In what follows, we require the centerpoint to belong to the set $S$. In this case, we cannot guarantee that there always exists a $\frac{1}{4}$-deep point in $S$. However, we can show that $S$ always contains a $\frac{1}{8}$-centerpoint.

Theorem 1 Any set of $n$ points in the plane has an element with opposite-quadrant depth at least $n / 8$. Moreover, such a point can be found in $O(n)$ time.

The proof is inspired by the "catline" of Hubert and Rousseuw [6].

Somewhat surprisingly, Theorem 1 is essentially tight.

Theorem 2 For infinitely many values of $n$, there are $n$-element sets $S_{n}$ with $\max _{p \in S_{n}} \operatorname{opp}\left(p, S_{n}\right)=$ $(n+4) / 8$.

If the points of $S$ are in convex position, that is, if they form the vertex set of a convex polygon, we can also guarantee the existence of a $\frac{1}{4}$-centerpoint in $S$. In fact, this statement remains true under the weaker condition that $S$ is an exposed set, that is, for any $p \in S$, at least one of the four quadrants $\mathrm{NW}(p), \mathrm{NE}(p), \mathrm{SE}(p)$, and $\mathrm{SW}(p)$ contains no element of $S$ in its interior. With a little twist of terminology, in this case we say that such a quadrant is empty (although it necessarily contains the point $p$ and may perhaps contain some other boundary points that belong to $S$ ).

Theorem 3 Any exposed set of $n$ points in the plane has an element with opposite-quadrant depth at least $n / 4$. Moreover, such a point can be found in $O(n)$ time.

The above notions are closely related to half-space depth, multivariate regression depth, and other measures of statistical depth [7]. For instance, the half-space depth of a point $p$ with respect to an $n$-element set $S$ in $d$-space is the minimum number of points of $S$ covered by a closed halfspace containing $p$. It is well-known that one can always find a point of half-space depth at least $\left\lfloor\frac{n}{d+1}\right\rfloor$ with respect to $S[2]$. In sharp contrast to the opposite-quadrant depth, however, now we cannot guarantee that $S$ has an element of large half-space depth. Indeed, if the points of $S$ are in strictly convex position, none of them has half-space depth larger than one.

\section{Proofs of Theorems 1 and 3}

First, notice that having points on the same vertical or horizontal line does not create a problem, since the quadrants were defined to be closed. In fact, we can slightly perturb our original point set $S$ by assigning to each element $p_{i} \in S$ a vector $\mathbf{v}_{i}=\left(i, i^{2}\right)$. Then there exists a sufficiently small $\varepsilon>0$ such that the modified sets $S_{\lambda}=\left\{p_{i}+\lambda \mathbf{v}_{i}\right\}$ are in general position for any $\lambda \in(0, \varepsilon]$, in the sense that no pair of points have the same $x$ or $y$ coordinate. Consequently, varying $\lambda$ in this small interval will not change the horizontal or vertical order of the elements of $S_{\lambda}$. If our theorems hold for sets in general position, we can apply them to $S_{\varepsilon}$, and find an $\alpha$-centerpoint $p_{i}+\varepsilon \mathbf{v}_{i}$. Then $p_{i}+\lambda \mathbf{v}_{i}$ is $\alpha$-deep with respect to $S_{\lambda}$ for all $\lambda \in(0, \varepsilon]$. Bringing back our points to their original positions in $S$ only adds points on the boundary of the two opposite quadrants of $p_{i}$. Therefore, $p_{i}$ is also an $\alpha$-centerpoint for $S$. 
Thus, from now on we assume that $S$ is in general position. To simplify the presentation, we also assume that $n=|S|$ is divisible by 8 , so that we do not have to deal with upper and lower integer parts. The proof of Theorem 1 proceeds by first splitting the set into three parts by two vertical lines, not passing through any element of $S$, such that in the left and right half-planes there are precisely $n / 4$ points and the vertical strip in the middle contains $n / 2$ points. Next, we find a horizontal line, not passing through any point of $S$, that divides $S$ into two equal halves. In this way, we obtain a $3 \times 2$ "grid," whose six regions will be called "cells." We say that a cell is heavy if it contains at least as many points as the other cell in the same column, that is, the cell below or above it. Note that if every cell in a row is heavy, then each of them contains precisely as many elements as the cell below or above it. Otherwise, there could not be $n / 2$ points in the other row. Thus, in this case, all six cells must be heavy. Therefore, we can always pick one heavy cell per column so that not all of them belong to the same row. We distinguish two cases up to symmetries (reflections about the coordinate axes).

(a) Bitonic configuration: The upper cells $H_{1}$ and $H_{3}$ of the first and third columns, respectively, and the lower cell $\mathrm{H}_{2}$ of the second column are all heavy. (See Figure 1(a).)

(b) Monotone configuration: The upper cells $H_{1}$ and $H_{2}$ of the first two columns and the lower cell $H_{3}$ of the third column are all heavy. (See Figure 1(b).)

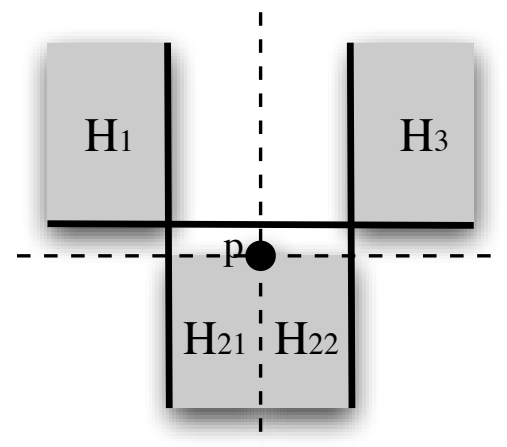

(a) Bitonic configuration

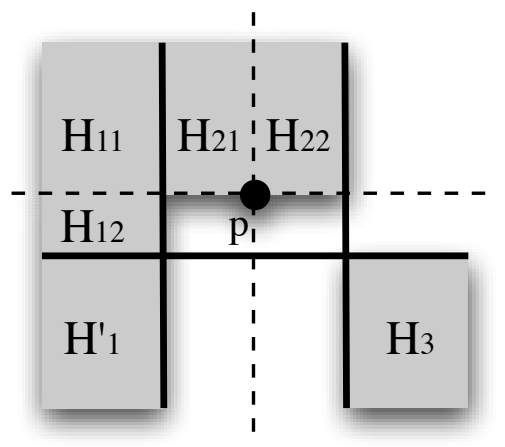

(b) Monotone configuration

Figure 1: Proof of Theorem 1. We let $H_{1}^{\prime}$ be the lower cell in the left column, $H_{11}:=H_{1} \cap \mathrm{NW}(p)$, $H_{12}:=H_{1} \cap \mathrm{SW}(p), H_{21}:=H_{2} \cap \mathrm{NW}(p)$, and $H_{22}:=H_{2} \cap \mathrm{NE}(p)$. Case (a): $H_{1}, H_{3}$, and one of $H_{21}$ or $H_{22}$ have $\geq n / 8$ points. Case (b): $H_{3}$ and one of $\mathrm{NW}(p)$ or $\mathrm{SW}(p)$ have $\geq n / 8$ points. If $\mathrm{NW}(p)$ does not, both $\mathrm{SW}(p)$ and $H_{22}$ have $\geq n / 8$ points.

First we prove Theorem 1 for bitonic configurations. Let $p$ be the highest point of the lower (heavy) cell $\mathrm{H}_{2}$ of the middle column. The quadrants $\mathrm{NW}(p)$ and $\mathrm{NE}(p)$ contain the heavy cells $H_{1}$ and $H_{3}$, therefore both of them have at least $n / 8$ points of $S$. All the at least $n / 4$ points in the heavy cell $\mathrm{H}_{2}$ that belong to $S$ lie in the half-plane $\mathrm{SW}(p) \cup \mathrm{SE}(p)$. Thus, at least one of the two quadrants, $\mathrm{SW}(p)$ or $\mathrm{SE}(p)$, must contain at least $n / 8$ points. This quadrant together with the opposite one forms a good pair, concluding the proof for bitonic configurations.

Next we establish Theorem 1 for monotone configurations. We have to use a slightly different argument, because we have no information about the relative vertical order of the points of $H_{1}$ and $H_{2}$. Choose $p$ to be the lowest point in $S$ that belongs to $H_{2}$, the upper cell in the middle column. Since $\mathrm{SE}(p)$ contains the heavy cell $H_{3}$, it has at least $n / 8$ points of $S$. If $\mathrm{NW}(p)$, the 
quadrant opposite to $\mathrm{SE}(p)$, also has at least $n / 8$ points, we are done. Otherwise, the number of points in $\mathrm{SW}(p)$ must exceed $n / 8$, because $\mathrm{NW}(p) \cup \mathrm{SW}(p)$ covers the $n / 4$ points in the first column. Moreover, if $\mathrm{NW}(p)$ has fewer than $n / 8$ points, then there must be more than $n / 8$ points in $\operatorname{NE}(p) \cap H_{2}$. Therefore, in this case both quadrants $\operatorname{SW}(p)$ and $\operatorname{NE}(p)$ have $n / 8$ points.

All of the above steps can be performed in linear time, first using a linear-time selection algorithm [1] to find the vertical lines with the properties described in the proof above, and the halving horizontal line, and finally performing several passes through the points to determine whether one has a bitonic or monotone configuration and to find the highest and lowest point in the appropriate cell. Hence one can find a point of depth at least $n / 8$ from an arbitrary point set in $O(n)$ time. This completes the proof of Theorem 1.

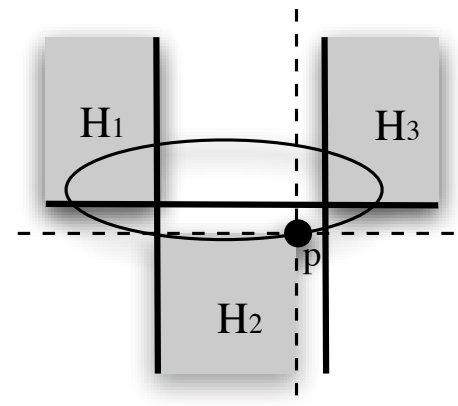

(a) Bitonic configuration

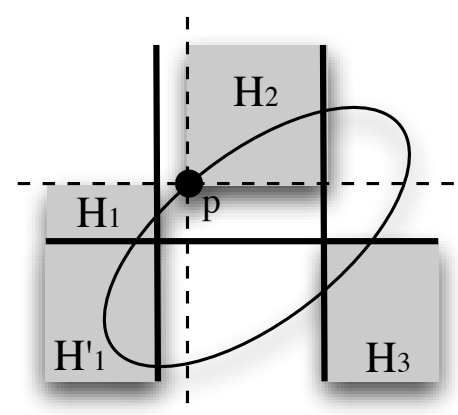

Monotone configuration

(c) $\mathrm{NW}(\mathrm{p})$ is empty.

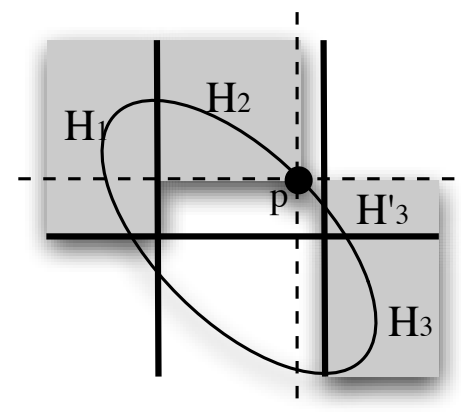

Monotone configuration

(b) $\mathrm{NE}(\mathrm{p})$ is empty.

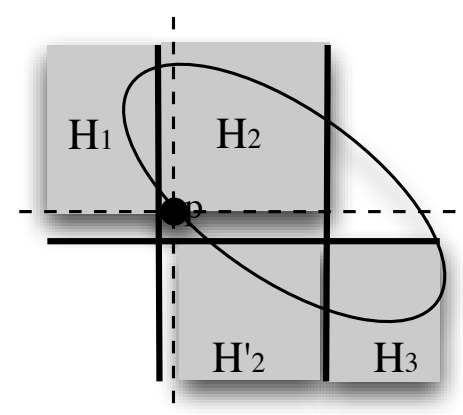

Monotone configuration

(d) SW(p) is empty.

Figure 2: Proof of Theorem 3 when all points belong to the convex curve shown. For each $i=1,2,3$, we let $H_{i}^{\prime}$ be the complement of $H_{i}$ in column $i$. Case (a): if $\mathrm{SE}(p)$ is empty, then $\mathrm{SW}(p)$ contains all points in $H_{2}$ and $\mathrm{NE}(p)$ contains all points in the last column. Case (b): if $\mathrm{NE}(p)$ is empty, then $\mathrm{NW}(p)$ contains all the $\geq n / 4$ points of $H_{2}$, and $\operatorname{SE}(p) \supseteq H_{3} \cup H_{3}^{\prime}$ contains all the $n / 4$ points of the right column; (c) if $\mathrm{NW}(p)$ is empty, then the situation is symmetric; and (d) if $\mathrm{SW}(p)$ is empty, then $\mathrm{NW}(p)$ contains all the $n / 4$ points in the left column and $\mathrm{SE}(p)$ contains all the $n / 2$ points in the bottom row.

Now we prove Theorem 3. We use the same configurations as for Theorem 1. (See Figure 2.) We want to use the assumption that every point $p \in S$ is "exposed," that is, one of the four quadrants determined by $p$ is "empty."

We first prove Theorem 3 for bitonic configurations. As in the proof of Theorem 1 , let $p \in S$ 
be the highest point in $H_{2}$. Observe that neither $\mathrm{NE}(p)$ nor $\mathrm{NW}(p)$ can be empty since each of them contains a heavy cell $\left(H_{1}\right.$ or $\left.H_{3}\right)$. Suppose without loss of generality that $\mathrm{SE}(p)$ is empty (see Figure 2(a)). Then $p$ is not only the highest, but also the rightmost point in its cell. Hence, $\mathrm{SW}(p)$ contains all at least $n / 4$ points in $H_{2}$, while $\mathrm{NE}(p)$ contains all $n / 4$ elements of the last column.

It remains to establish Theorem 3 for monotone configurations. Again, let $p$ be the lowest element of $S$ in $H_{2}$. Now one of the four quadrants at $p$ must be empty. Observe that $\operatorname{SE}(p)$ covers the heavy cell $\mathrm{H}_{3}$, so it is not empty. If $N E(p)$ is empty, then $N W(p)$ contains all the at least $n / 4$ points in $H_{2}$, and $S E(p)$ contains all the $n / 4$ points of $S$ in the third column, so we are done (Figure 2(b)). By symmetry, if $N W(p)$ is empty, both $\mathrm{NE}(p)$ and $\mathrm{SW}(p)$ contain at least $n / 4$ points (Figure 2(c)). Finally, if $S W(p)$ is empty, then $N W(p)$ contains all the $n / 4$ points of $S$ in the first column, and $S E(p)$ contains all the $n / 2$ points of $S$ in the second row, so we are also done in this case (Figure 2(d)). The running time analysis for finding such a point $p$ of depth at least $n / 4$ is the same as for Theorem 1 . This completes the proof of Theorem 3 .

\section{Proof of Theorem 2}

Let $\varepsilon$ be a small positive number, let $\theta$ be the counterclockwise rotation by $\frac{\pi}{2}$ about the origin, and let $S_{1}$ denote the set consisting of the following four points:

$$
T:=(-2 \varepsilon, 1), \quad L:=(-1,-2 \varepsilon), \quad B:=(2 \varepsilon,-1), \quad R:=(1,2 \varepsilon) .
$$

Setting $T_{1}=\{T\}, L_{1}=\{L\}, B_{1}=\{B\}, R_{1}=\{R\}$, we have $S_{1}=T_{1} \cup L_{1} \cup B_{1} \cup R_{1}$. Now let $k>1$, and suppose that $T_{k-1}, L_{k-1}, B_{k-1}, R_{k-1}$, and $S_{k-1}=T_{k-1} \cup L_{k-1} \cup B_{k-1} \cup R_{k-1}$ have already been defined. Let

$$
\begin{aligned}
T_{k} & :=T_{k-1} \oplus\left(\varepsilon^{k} \Delta\right), \\
L_{k} & :=L_{k-1} \oplus\left(\varepsilon^{k} \theta(\Delta)\right), \\
B_{k} & :=B_{k-1} \oplus\left(\varepsilon^{k} \theta^{2}(\Delta)\right), \\
R_{k} & :=R_{k-1} \oplus\left(\varepsilon^{k} \theta^{3}(\Delta)\right),
\end{aligned}
$$

where $\Delta=\{L, B, R\}$ and $\oplus$ stands for the Minkowski sum. Finally, define $S_{k}:=T_{k} \cup L_{k} \cup B_{k} \cup R_{k}$. See Figure 3.

Obviously, we have $\left|S_{k}\right|=4 \cdot 3^{k-1}$, and the set $S_{k}$ is invariant under the rotation $\theta$.

Clearly, $\operatorname{opp}\left(p, S_{1}\right)=1$ holds for every $p \in S_{1}$. It is easy to show by induction on $k$ that

$$
\left|\mathrm{NW}(p) \cap T_{k}\right| \leq \frac{3^{k-1}+1}{2},
$$

for every $p \in T_{k}$. For $k=1$ there is nothing to prove. Let $k>1$, and suppose that we have already verified the statement for $k-1$. Write $p \in T_{k}$ in the form $q \oplus\left(\varepsilon^{k} r\right)$, for some $q \in T_{k-1}$ and $r \in \Delta$. Using the induction hypothesis, we obtain that $\mathrm{NW}(q)$, and hence also $\mathrm{NW}(p)$, contains at most $\frac{3^{k-2}+1}{2}-1$ points of $T_{k-1}$ in its interior, each giving rise to precisely three points of $T_{k}$. In addition, by the construction of $\Delta$, the quadrant $\mathrm{NW}(p)$ contains at most two elements of the triple $\left\{q \oplus\left(\varepsilon^{k} r\right) \mid r \in \Delta\right\}$. That is, we have

$$
\left|\mathrm{NW}(p) \cap T_{k}\right| \leq 3\left(\frac{3^{k-2}+1}{2}-1\right)+2=\frac{3^{k-1}+1}{2},
$$




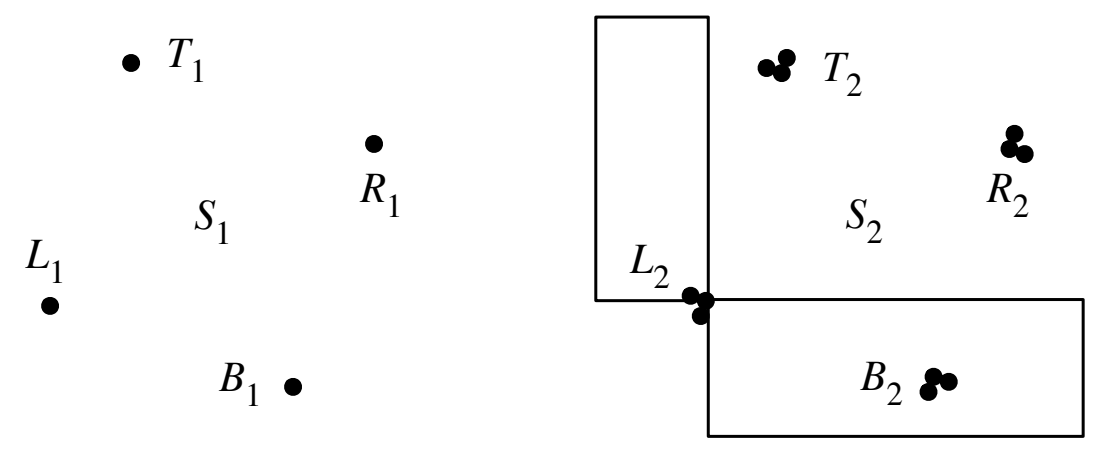

Figure 3: The construction for the proof of Theorem 2. The set $S_{1}$ of four points (right), and the set $S_{2}$ of 12 points has opposite-quadrant depth 2 , shown with a pair of opposite quadrants with 2 and 5 points, respectively.

as required. Analogously, we obtain

$$
\left|\mathrm{NE}(p) \cap T_{k}\right| \leq \frac{3^{k-1}+1}{2}
$$

for every $p \in T_{k}$, because $N E(p)$ also contains at most two elements of the triple $\left\{q \oplus\left(\varepsilon^{k} r\right) \mid r \in \Delta\right\}$.

These two bounds imply that

$$
\operatorname{opp}\left(p, S_{k}\right) \leq \frac{3^{k-1}+1}{2}=\frac{\left|S_{k}\right|+4}{8},
$$

for every $k \geq 1$ and $p \in T_{k}$. The easy verification of the fact that equality can be attained here is left to the reader. Since the construction is symmetric under the rotation theta, we obtain that the same bound is true for every $p \in S_{k}$. This completes the proof of Theorem 2 .

\section{Concluding remarks}

The notion of opposite-quadrant depth arose in connection with conflict-free coloring problems $[4,5]$. Take $n$ points in the plane in general position and connect two of them by an edge if the smallest axis-parallel rectangle containing them covers no other point of the set. Is it true that this graph contains a large independent set?

Let $f(n)$ denote the size of the largest independent set whose existence can always be guaranteed and suppose for a moment that there exists a point $p$ whose opposite-quadrant depth is $n / 4$. That is, $\mathrm{NW}(p)$ and $\mathrm{SE}(p)$, say, both contain at least $n / 4$ points. The point $p$ guarantees that no pair of points lying in opposite quadrants are connected by an edge. Thus, after recursively finding independents sets in the subgraphs induced by $\mathrm{NW}(p)$ and $\mathrm{SE}(p)$, their union is always an independent set in the whole graph. Thus, we would have $f(n) \geq 2 f(n / 4)-1$, implying that $f(n)=\Omega(\sqrt{n})$. Unfortunately, Theorem 1 yields only $f(n)=\Omega\left(n^{1 / 3}\right)$, and, by Theorem 2 , this method cannot lead to a better bound. Nevertheless, by an easy application of a lemma of Erdős and Szekeres [3], we obtain $f(n)=\Omega(\sqrt{n})$, and this bound can be slightly improved [9]. It is not known whether $f(n)=o(n)$. 
The natural extension of opposite-quadrant depth to opposite-orthant depth in three dimensions seems to lead to interesting questions. Curiously, the opposite-orthant depth of the intersection point of three halving planes can be zero. For instance, place $n / 4$ points in small neighborhoods of the points $(1,1,1),(1,-1,-1),(-1,1,-1)$ and $(-1,-1,1)$. However, it is not hard to see that any set of $n$ points in three-dimensional space has an element of opposite-orthant depth at least $c n$, where $c>0$ is an appropriate constant. Indeed, it follows by the repeated application of the lemma of Erdős and Szekeres [3] mentioned earlier that any 9-tuple of points in three-dimensional space contains a triple $(p, q, r)$ such that $p$ and $r$ belong to opposite orthants with respect to $q$. Clearly, the number of distinct triples that can be obtained in this way is at least

$$
\frac{\left(\begin{array}{l}
n \\
9
\end{array}\right)}{\left(\begin{array}{c}
n-3 \\
6
\end{array}\right)}=\frac{\left(\begin{array}{l}
n \\
3
\end{array}\right)}{\left(\begin{array}{l}
9 \\
3
\end{array}\right)} .
$$

Therefore, there exists a point $q^{*}$ which plays the role of the "middle" element in at least

$$
\frac{1}{n} \cdot \frac{\left(\begin{array}{l}
n \\
3
\end{array}\right)}{\left(\begin{array}{l}
9 \\
3
\end{array}\right)}
$$

such triples. At least one quarter of these triples have the same type, in the sense that the other two elements belong to the same pair of opposite orthants. That is, the opposite-orthant depth of $q^{*}$ is at least

$$
\frac{1}{4 n^{2}} \cdot \frac{\left(\begin{array}{l}
n \\
3
\end{array}\right)}{\left(\begin{array}{l}
9 \\
3
\end{array}\right)}=\Omega(n)
$$

as required. The same argument works in higher dimensions. For similar results, consult [8].

An alternate extension of $\operatorname{opp}(p, S)$ to three dimensions is $\max _{\sigma, \tau} \min (|S \cap \sigma|,|S \cap \tau|)$, over all pairs $\sigma, \tau$ of opposite quadrants determined by a pair of axis-aligned planes. There are now six pairs of opposite quadrants. With this definition, all planar lower bounds remain valid in three dimensions, but they are not necessarily tight.

Acknowledgements Thanks to Boris Aronov, Joshua Cooper, Raghavan Dhandapani, Jeff Erickson, John Iacono, and Rados̆ Radoičić for discussions and comments.

\section{References}

[1] T. Cormen, C. Leiserson, R. Rivest, and C. Stein. Introduction to Algorithms (2nd ed.). MIT Press, Cambridge, MA, 2001.

[2] H. Edelsbrunner. Algorithms in Combinatorial Geometry. Springer, Berlin, 1987.

[3] P. Erdős and G. Szekeres. A combinatorial problem in geometry. Compositio Math. 2:463-470, 1935.

[4] G. Even, Z. Lotker, D. Ron, and S. Smorodinsky. Conflict-free colorings of simple geometric regions with applications to frequency assignments in cellular networks. SIAM J. Comput. 33(1):94-136, 2003.

[5] S. Har-Peled and S. Smorodinsky. Conflict-free colorings of points and simple regions in the plane. Discrete and Comput. Geom. 34:47-70, 2005. 
[6] M. Hubert and P. Rousseeuw. The catline for deep regression. J. Multivariate Analysis 66(2):270-296, 1998.

[7] R. Liu, J. Parelius, and K. Singh. Multivariate analysis by data depth: Descriptive statistics, graphics and inference. Ann. Stat. 27:783-858, 1999.

[8] J. Pach. A remark on transversal numbers. The mathematics of Paul Erdös, II, Algorithms Combin. 14:310-317, Springer, Berlin, 1997.

[9] J. Pach and G. Tóth. Conflict-free colorings. Discrete and Computational Geometry, The Goodman-Pollack Festschrift. Springer Verlag, Heidelberg, 2003. 665-671. 